\title{
EQUIVALENCE OF THE CONVERGENCE OF MANN \\ ITERATION WITH MODIFIED ERRORS AND \\ ISHIKAWA ITERATION WITH MODIFIED ERRORS \\ FOR THE CLASS OF UNIFORMLY CONTINUOUS, STRONGLY PSEUDOCONTRACTIVE MAPS
}

\section{\|\|}

\author{
G. V. R. BABU AND M. V. R. KAMESWARI
}

\begin{abstract}
We prove that the convergence of the Mann iteration with modified errors is equivalent to the convergence of the Ishikawa iteration with modified errors for the class of uniformly continuous and strongly pseudocontractive maps. Our results improve the results of Soltuz [14] and extend the results of Rhoades and Soltuz [12] to the iterations with modified errors.
\end{abstract}

\section{Introduction}

Throught this paper, $E$ denotes a real Banach space and $E^{*}$, the dual of $E$; and $I$ denotes the identity operator on $E$.

We denote by $J$, the duality map from $E$ to $2^{E^{*}}$ defined by

$$
J(x)=\left\{f^{*} \in E^{*}:\left\langle x, f^{*}\right\rangle=\|x\|^{2}=\|f\|^{2}\right\},
$$

where $\langle\cdot, \cdot\rangle$ is the generalized duality pairing of $E$ and $E^{*}$.

A map $T$ from $E$ to $E$ is said to be strongly pseudocontractive if there exists $t>1$ such that

$$
\|x-y\| \leq\|(1+r)(x-y)-r t(T x-T y)\|
$$

for all $x, y$ in $E$ and $r>0$.

If $t=1$, then $T$ is called a pseudocontrative map.

A mapping $T$ with domain $D(T)$ and range $R(T)$ in $E$ is called accretive if for all $x, y \in D(T)$, there exists $t>1$ such that

$$
\|x-y\| \leq\|(x-y)+k(T x-T y)\|
$$

for every $k>0$, and $T$ is said to be strongly accretive if there is a positive constant $k$ such that $T-k I$ is accretive.

Received September 30, 2005.

2000 Mathematics Subject Classification. 47H10, 54H25.

Key words and phrases. Mann iteration, Ishikawa iteration, Mann iteration with modified errors, uniformly continuous maps, strongly pseudocontractive maps, strongly accretive operators. 
The pseudocontractive maps are characterized by the fact that the mapping $T$ is pseudocontractive if and only if $I-T$ is accretive.

As a consequence of the result of Kato [9], $T$ is pseudocontractive if and only if for all $x, y \in D(T)$, there exists $j(x-y) \in J(x-y)$ such that $\langle(I-T) x-(I-T) y, j(x-y)\rangle \geq 0$. that

Furthermore, $T$ is strongly pseudocontractive if and only if there exists a $k>0$ such

$$
\langle(I-T) x-(I-T) y, j(x-y)\rangle \geq k\|x-y\|^{2}
$$

for all $x, y$ in $D(T)$.

Also, from Bogin [1], it follows that the inequality (1.1.3) inplies that $T$ is strongly pseudocontractive with $k \in(0,1)$ if and only if for all $x, y \in D(T)$ the following inequality holds:

$$
\|x-y\| \leq\|x-y+s[(I-T-k I) x-(I-T-k I) y]\|
$$

for all $s>0$.

According to Deimling [5], if $K$ is a closed convex subset of $E$ and $T: K \rightarrow K$ is continuous, strongly pseudocontractive then $T$ has a unique fixed point in $K$. Further, it is proved ([6], Theorem 13.1, p.125) that if $T: E \rightarrow E$ is continuous and strongly accretive then $T$ is surjective; i.e., for a give $f \in E$ the equation $T x=f$ has a unique solution.

For a selfmap $T$ of $K$, we denote $F(T)$, the fixed point set of $T$.

Let $K$ be a nonempty convex subset of $E$ and $T$ a selfmap of $K$. For $u_{0} \in K$, the sequence $\left\{u_{n}\right\}_{n=0}^{\infty} \subset K$ defined by

$$
u_{n+1}=\left(1-\alpha_{n}\right) u_{n}+\alpha_{n} T u_{n}, \quad n=0,1,2, \ldots
$$

is called the Mann iteration, where $\left\{\alpha_{n}\right\}$ is a sequence of positive numbers in $(0,1)$ satisfying

$$
\lim _{n \rightarrow \infty} \alpha_{n}=0 \text { and } \Sigma \alpha_{n}=\infty .
$$

For $x_{0} \in K$, the sequence $\left\{x_{n}\right\}_{n=0}^{\infty} \subset K$ defined by

$$
\begin{aligned}
x_{n+1} & =\left(1-\alpha_{n}\right) x_{n}+\alpha_{n} T y_{n}, \\
y_{n} & =\left(1-\beta_{n}\right) x_{n}+\beta_{n} T x_{n}, \quad n=0,1,2, \ldots,
\end{aligned}
$$

is called the Ishikawa iteration, where $\left\{\alpha_{n}\right\}$ and $\left\{\beta_{n}\right\}$ are sequences of positive numbers in $(0,1)$ satisfying

$$
\lim _{n \rightarrow \infty} \alpha_{n}=0, \quad \lim _{n \rightarrow \infty} \beta_{n}=0 \text { and } \Sigma \alpha_{n}=\infty .
$$

In recent years, Mann and Ishikawa iterations have been studied extensively by several authors $[2,3,4,7,13]$ for the class of strongly pseudocontractive operators.

Rhoades and Soltuz [12] studied the equivalence between the convergences of Mann and Ishikawa iterations and established the following theorem.

Theorem 1.1. Let $K$ be a closed, convex subset of $E$ and $T$ a Lipschitz, strongly pseudocontractive selfmap of $K$. Consider the Mann and Ishikawa iterations with the same initial point as in (1.1.5) and (1.1.7), $\left\{\alpha_{n}\right\}$ and $\left\{\beta_{n}\right\}$ are sequences of positive numbers in $(0,1)$ satisfying (1.1.8). Then the following are equivalent: 
(1) The Mann iteration (1.1.5) converges to $x^{*} \in F(T)$.

(2) The Ishikawa iteration (1.1.7) converges to $x^{*} \in F(T)$.

In 1995, Liu [10] introduced iterations with errors as follows:

For a nonempty convex subset $K$ of $E$ and $T: K \rightarrow E$, for $u_{0} \in K$, the sequence $\left\{u_{n}\right\}_{n=0}^{\infty} \subset K$ defined by

$$
u_{n+1}=\left(1-\alpha_{n}\right) u_{n}+\alpha_{n} T u_{n}+e_{n}, \quad n=0,1,2, \ldots
$$

where (i) $\left\{\alpha_{n}\right\}$ is a sequence of positive numbers in $(0,1)$ satisfying (1.1.6) and (ii) $\Sigma\left\|e_{n}\right\|<\infty$, is called the Mann iteration with errors.

For $x_{0} \in K$, the sequence $\left\{x_{n}\right\}_{n=0}^{\infty} \subset K$ defined by

$$
\begin{aligned}
x_{n+1} & =\left(1-\alpha_{n}\right) x_{n}+\alpha_{n} T y_{n}+p_{n}, \\
y_{n} & =\left(1-\beta_{n}\right) x_{n}+\beta_{n} T x_{n}+q_{n}, \quad n=0,1,2, \ldots
\end{aligned}
$$

where (i) $\left\{\alpha_{n}\right\}$ and $\left\{\beta_{n}\right\}$ are sequences of positive numbers satisfying (1.1.8) and (ii) $\Sigma\left\|p_{n}\right\|<\infty, \Sigma\left\|q_{n}\right\|<\infty$, is called the Ishikawa iteration with errors.

Soltuz 14 obtained the following result, to establish the equivalence between the Mann and Ishikawa iterations with errors.

Theorem 1.2. Let $K$ be a nonempty, bounded, convex and closed subset of $E$ and $T: K \rightarrow E$ a strongly pseudocontractive and Lipschitzian map with Lipschitz constant $L>1$ with $T(K)$ bounded and $F(T) \neq \varnothing$. If $x_{0}=u_{0} \in K$, then the following assertions are equivalent:

(1) The Mann iteration with errors $(A)$ converges to $x^{*} \in F(T)$.

(2) The Ishikawa iteration with errors $(B)$ converges to $x^{*} \in F(T)$.

In particular, the conditions $\Sigma\left\|e_{n}\right\|<\infty, \Sigma\left\|p_{n}\right\|<\infty$ and $\Sigma\left\|q_{n}\right\|<\infty$ imply that the error terms in (A) and (B) tend to zero as $n \rightarrow \infty$. This is incompatible with the randomness of the occurrence of errors. Afterwards, in 1998, Xu [15] introduced a more satisfactory error terms in iterations in the following way.

Let $K$ be a nonempty convex subset of $E$ and $T: K \rightarrow K$ a mapping. For given $u_{0} \in E$, the sequence $\left\{u_{n}\right\}_{n=0}^{\infty} \subset K$ defined by

$$
u_{n+1}=a_{n} u_{n}+b_{n} T u_{n}+c_{n} e_{n}, \quad n=0,1,2, \ldots
$$

where $\left\{e_{n}\right\}$ is a bounded sequence in $K$ and $\left\{a_{n}\right\},\left\{b_{n}\right\},\left\{c_{n}\right\}$ are sequences in $(0,1)$ such that $a_{n}+b_{n}+c_{n}=1$, for all $n \geq 0$, is called the Mann iteration with modified errors.

For a given $x_{0} \in E$, the sequence $\left\{x_{n}\right\}_{n=0}^{\infty} \subset K$ defined by

$$
\begin{aligned}
x_{n+1} & =a_{n} x_{n}+b_{n} T y_{n}+c_{n} p_{n}, \\
y_{n} & =a_{n}^{\prime} x_{n}+b_{n}^{\prime} T x_{n}+c_{n}^{\prime} q_{n}, \quad n=0,1,2, \ldots
\end{aligned}
$$


where $\left\{p_{n}\right\},\left\{q_{n}\right\}$ are bounded sequences in $K$ and $\left\{a_{n}\right\},\left\{b_{n}\right\},\left\{c_{n}\right\},\left\{a_{n}^{\prime}\right\},\left\{b_{n}^{\prime}\right\}$ and $\left\{c_{n}^{\prime}\right\}$ are sequences in $(0,1)$ such that $a_{n}+b_{n}+c_{n}=1=a_{n}^{\prime}+b_{n}^{\prime}+c_{n}^{\prime}=1$, for all $n \geq 0$, is called the Ishikawa iteration with modified errors. If $K$ is bounded then the sequences $\left\{p_{n}\right\},\left\{q_{n}\right\}$ and $\left\{e_{n}\right\}$ in the iterations can be arbitrarily.

In 1998, Chidume [3] established the following convergence theorems.

Theorem 1.3. Let $K$ be a closed, convex and bounded subset of $E, T: K \rightarrow K a$ uniformly continuous and strongly pseudocontractive mapping. For a given $x_{0}, p_{0}, q_{0} \in K$, the sequence $\left\{x_{n}\right\}_{n=0}^{\infty}$ defined by

$$
\begin{aligned}
x_{n+1} & =a_{n} x_{n}+b_{n} T y_{n}+c_{n} p_{n}, \\
y_{n} & =a_{n}^{\prime} x_{n}+b_{n}^{\prime} T x_{n}+c_{n}^{\prime} q_{n}, \quad n=0,1,2, \ldots
\end{aligned}
$$

where $\left\{p_{n}\right\},\left\{q_{n}\right\}$ are arbitrary sequences in $K$ and $\left\{a_{n}\right\},\left\{b_{n}\right\},\left\{c_{n}\right\},\left\{a_{n}^{\prime}\right\},\left\{b_{n}^{\prime}\right\}$ and $\left\{c_{n}^{\prime}\right\}$ are sequences in $(0,1)$ satisfying

(i) $a_{n}+b_{n}+c_{n}=1=a_{n}^{\prime}+b_{n}^{\prime}+c_{n}^{\prime}=1$, for all $n \geq 0$,

(ii) $\Sigma b_{n}=\infty$,

(iii) $\Sigma c_{n}<\infty$, and

(iv) $\lim _{n \rightarrow \infty} b_{n}=\lim _{n \rightarrow \infty} b_{n}^{\prime}=\lim _{n \rightarrow \infty} c_{n}^{\prime}=0$.

Then $\left\{x_{n}\right\}_{n=0}^{\infty}$ converges strongly to $x^{*} \in F(T)$.

Theorem 1.4. Let $E, K$ and $T$ be as in Theorem 1.3. For a given $x_{0}, p_{0} \in K$, the sequence $\left\{x_{n}\right\}_{n=0}^{\infty}$ defined by

$$
x_{n+1}=a_{n} x_{n}+b_{n} T x_{n}+c_{n} p_{n}, \quad n=0,1,2, \ldots
$$

where $\left\{p_{n}\right\}$ is an arbitrary sequence in $K$ and $\left\{a_{n}\right\},\left\{b_{n}\right\}$, and $\left\{c_{n}\right\}$ are sequences in $(0,1)$ satisfying

(i) $a_{n}+b_{n}+c_{n}=1$, for all $n \geq 0$,

(ii) $\Sigma b_{n}=\infty$,

(iii) $\Sigma c_{n}<\infty$, and

(iv) $\lim _{n \rightarrow \infty} b_{n}=0$.

Then $\left\{x_{n}\right\}_{n=0}^{\infty}$ converges strongly to $x^{*} \in F(T)$.

In this paper, we prove that the convergence of Mann iteration with modified errors and Ishikawa iteration with modified errors are equivalent for the class of uniformly continuous and strongly pseudocontractive maps. Also, we obtain the equivalence between the convergences of Mann and Ishikawa iterations with modified errors to the solution of the operator equation $A x=f$ (Corollary 2.5) and $x+T x=f$ (Corollary 2.6).

To prove our main results, we use the following lemma.

Lemma 1.5.(10]). Let $\rho_{n}$ be a non-negative sequence of reals satisfying

$$
\rho_{n+1} \leq\left(1-\lambda_{n}\right) \rho_{n}+\sigma_{n}+\gamma_{n},
$$


with $\left\{\lambda_{n}: n=0,1,2, \ldots\right\} \subset[0,1], \Sigma \lambda_{n}=\infty, \sigma_{n}=o\left(\lambda_{n}\right)$, and $\Sigma \gamma_{n}<\infty$. Then $\lim _{n \rightarrow \infty} \rho_{n}=0$.

\section{Main Results}

Theorem 2.1. Let $E, K$ and $T$ be as in Theorem 1.3. For a given $x_{0}, p_{0}, q_{0}, u_{0}$ and $e_{0} \in E$, we define the sequences $\left\{u_{n}\right\}_{n=0}^{\infty}$ and $\left\{x_{n}\right\}_{n=0}^{\infty}$ by

$$
u_{n+1}=a_{n} u_{n}+b_{n} T u_{n}+c_{n} e_{n}, \quad n=0,1,2,3, \ldots
$$

and

$$
\begin{aligned}
x_{n+1} & =a_{n} x_{n}+b_{n} T y_{n}+c_{n} p_{n}, \\
y_{n} & =a_{n}^{\prime} x_{n}+b_{n}^{\prime} T x_{n}+c_{n}^{\prime} q_{n}, \quad n=0,1,2, \ldots
\end{aligned}
$$

where $\left\{p_{n}\right\},\left\{q_{n}\right\}$ and $\left\{e_{n}\right\}$ are arbitrary sequences in $K$ and $\left\{a_{n}\right\},\left\{b_{n}\right\},\left\{c_{n}\right\},\left\{a_{n}^{\prime}\right\}$, $\left\{b_{n}^{\prime}\right\}$ and $\left\{c_{n}^{\prime}\right\}$ are sequences in $(0,1)$ satisfying

(i) $a_{n}+b_{n}+c_{n}=1=a_{n}^{\prime}+b_{n}^{\prime}+c_{n}^{\prime}=1$, for all $n \geq 0$,

(ii) $\Sigma b_{n}=\infty$,

(iii) $\Sigma c_{n}<\infty$, and

(iv) $\lim _{n \rightarrow \infty} b_{n}=\lim _{n \rightarrow \infty} b_{n}^{\prime}=\lim _{n \rightarrow \infty} c_{n}^{\prime}=0$.

If $u_{0}=x_{0} \in K$, then the following are equivalent:

(1) The Mann iteration with modified errors $\left\{u_{n}\right\}$ defined by (2.1.1) converges to $x^{*} \in$ $F(T)$.

(2) The Ishikawa iteration with modified errors $\left\{x_{n}\right\}$ defined by (2.1.2) converges to $x^{*} \in F(T)$.

Proof. Set $\alpha_{n}=b_{n}+c_{n}$. From (2.1.2), we have

$$
x_{n+1}=\left(1-\alpha_{n}\right) x_{n}+\alpha_{n} T y_{n}-c_{n}\left(T y_{n}-p_{n}\right) \text {. }
$$

Thus

$$
\begin{aligned}
x_{n}= & \left(1+\alpha_{n}\right) x_{n+1}+\alpha_{n}(I-T-k I) x_{n+1}-(1-k) \alpha_{n} x_{n} \\
& +(2-k) \alpha_{n}^{2}\left(x_{n}-T y_{n}\right)+\alpha_{n}\left(T x_{n+1}-T y_{n}\right) \\
& +c_{n}\left[1+(2-k) \alpha_{n}\right]\left[T y_{n}-p_{n}\right] .
\end{aligned}
$$

Similarly from (2.1.1), we get

$$
\begin{aligned}
u_{n}= & \left(1+\alpha_{n}\right) u_{n+1}+\alpha_{n}(I-T-k I) u_{n+1}-(1-k) \alpha_{n} u_{n} \\
& +(2-k) \alpha_{n}^{2}\left(u_{n}-T u_{n}\right)+\alpha_{n}\left(T u_{n+1}-T u_{n}\right) \\
& +c_{n}\left[1+(2-k) \alpha_{n}\right]\left[T u_{n}-e_{n}\right] .
\end{aligned}
$$


Hence from (2.1.3) and (2.1.4), we have

$$
\begin{aligned}
x_{n}-u_{n}= & \left(1+\alpha_{n}\right)\left(x_{n+1}-u_{n+1}\right)+\alpha_{n}(I-T-k I) x_{n+1}-\alpha_{n}(I-T-k I) u_{n+1} \\
& -(1-k) \alpha_{n}\left(x_{n}-u_{n}\right)+(2-k) \alpha_{n}^{2}\left(x_{n}-T y_{n}-u_{n}+T u_{n}\right) \\
& +\alpha_{n}\left(T x_{n+1}-T y_{n}-T u_{n+1}+T u_{n}\right) \\
& +c_{n}\left[1+(2-k) \alpha_{n}\right]\left(T y_{n}-p_{n}-T u_{n}+e_{n}\right) .
\end{aligned}
$$

Now on taking norms on both sides, we have

$$
\begin{aligned}
\left\|x_{n}-u_{n}\right\| \geq & \|\left(1+\alpha_{n}\right)\left(x_{n+1}-u_{n+1}\right)+\alpha_{n}(I-T-k I) x_{n+1} \\
& -\alpha_{n}(I-T-k I) u_{n+1}\left\|-(1-k) \alpha_{n}\right\| x_{n}-u_{n} \| \\
& -(2-k) \alpha_{n}^{2}\left\|x_{n}-T y_{n}-u_{n}+T u_{n}\right\| \\
& -\alpha_{n}\left\|T x_{n+1}-T y_{n}-T u_{n+1}+T u_{n}\right\| \\
& -c_{n}\left[1+(2-k) \alpha_{n}\right]\left\|T y_{n}-p_{n}-T u_{n}+e_{n}\right\| .
\end{aligned}
$$

By using the inequality (1.1.4) with $x=x_{n+1}, y=u_{n+1}$ and $s=\frac{\alpha_{n}}{1+\alpha_{n}}$ we get

$$
\begin{aligned}
\left\|x_{n}-u_{n}\right\| \geq & \left(1+\alpha_{n}\right)\left\|x_{n+1}-u_{n+1}\right\|-(1-k) \alpha_{n}\left\|x_{n}-u_{n}\right\| \\
& -(2-k) \alpha_{n}^{2}\left\|x_{n}-T y_{n}-u_{n}+T u_{n}\right\| \\
& -\alpha_{n}\left\|T x_{n+1}-T y_{n}-T u_{n+1}+T u_{n}\right\| \\
& -c_{n}\left[1+(2-k) \alpha_{n}\right]\left\|T y_{n}-p_{n}-T u_{n}+e_{n}\right\| .
\end{aligned}
$$

Thus

$$
\begin{aligned}
\left(1+\alpha_{n}\right)\left\|x_{n+1}-u_{n+1}\right\| \leq & \left(1+(1-k) \alpha_{n}\right)\left\|x_{n}-u_{n}\right\| \\
& +(2-k) \alpha_{n}^{2}\left\|x_{n}-T y_{n}-u_{n}+T u_{n}\right\| \\
& +\alpha_{n}\left\|T x_{n+1}-T y_{n}-T u_{n+1}+T u_{n}\right\| \\
& +c_{n}\left[1+(2-k) \alpha_{n}\right]\left\|T y_{n}-p_{n}-T u_{n}+e_{n}\right\| .
\end{aligned}
$$

This implies

$$
\begin{aligned}
\left\|x_{n+1}-u_{n+1}\right\| \leq & \frac{\left(1+(1-k) \alpha_{n}\right)}{\left(1+\alpha_{n}\right)}\left\|x_{n}-u_{n}\right\|+(2-k) \alpha_{n}^{2}\left[\left\|x_{n}-T y_{n}\right\|+\left\|u_{n}-T u_{n}\right\|\right] \\
& +\alpha_{n}\left[\left\|T x_{n+1}-T y_{n}\right\|+\left\|T u_{n+1}-T u_{n}\right\|\right] \\
& +c_{n}\left[1+(2-k) \alpha_{n}\right]\left[\left\|T y_{n}-p_{n}\right\|+\left\|T u_{n}-e_{n}\right\|\right] \\
\leq & \left(1-k \alpha_{n}+\alpha_{n}^{2}\right)\left\|x_{n}-u_{n}\right\|+(2-k) \alpha_{n}^{2}\left[\left\|x_{n}-T y_{n}\right\|+\left\|u_{n}-T u_{n}\right\|\right] \\
& +\alpha_{n}\left[\left\|T x_{n+1}-T y_{n}\right\|+\left\|T u_{n+1}-T u_{n}\right\|\right] \\
& +c_{n}\left[1+(2-k) \alpha_{n}\right]\left[\left\|T y_{n}-p_{n}\right\|+\left\|T u_{n}-e_{n}\right\|\right] \\
\leq & \left(1-k \alpha_{n}\right)\left\|x_{n}-u_{n}\right\|+(3-k) \alpha_{n}^{2}\left\|x_{n}-T y_{n}\right\| \\
& +(2-k) \alpha_{n}^{2}\left\|u_{n}-T u_{n}\right\|+\alpha_{n}^{2}\left\|u_{n}-T y_{n}\right\| \\
& +\alpha_{n}\left[\left\|T x_{n+1}-T y_{n}\right\|+\left\|T u_{n+1}-T u_{n}\right\|\right] \\
& +2 c_{n} l_{n}\left[\left\|T y_{n}-p_{n}\right\|+\left\|T u_{n}-e_{n}\right\|\right]
\end{aligned}
$$


where $l_{n}=\left(1+(2-k) \alpha_{n}\right) \leq M_{1}$, with $M_{1}=3-k$. Thus

$$
\begin{aligned}
\left\|x_{n+1}-u_{n+1}\right\| \leq & \left(1-k \alpha_{n}\right)\left\|x_{n}-u_{n}\right\|+(4-k) \alpha_{n}^{2} D \\
& +(2-k) \alpha_{n}^{2}\left\|u_{n}-T u_{n}\right\| \\
& +\alpha_{n}\left[\left\|T x_{n+1}-T y_{n}\right\|+\left\|T u_{n+1}-T u_{n}\right\|\right] \\
& +2 c_{n} l_{n} D
\end{aligned}
$$

where $D$ is the diameter of $K$.

Write $\beta_{n}=b_{n}^{\prime}+c_{n}^{\prime}$ in $(2.1 .2)$, then we have

$$
y_{n}=\left(1-\beta_{n}\right) x_{n}+\beta_{n} T x_{n}-c_{n}^{\prime}\left(T x_{n}-q_{n}\right)
$$

Consider

$$
\begin{aligned}
\left\|x_{n+1}-y_{n}\right\| & \leq\left\|x_{n+1}-x_{n}\right\|+\left\|x_{n}-y_{n}\right\| \\
& \leq\left(\alpha_{n}+c_{n}\right) D+\left(\beta_{n}+c_{n}^{\prime}\right) D \rightarrow 0 \text { as } n \rightarrow \infty .
\end{aligned}
$$

Now by using uniform continuity of $T$, we get

$$
\left\|T x_{n+1}-T y_{n}\right\| \rightarrow 0 \text { as } n \rightarrow \infty \text {. }
$$

Since $T$ is uniformly continuous and strongly pseudocontractive on $K$, it follows by Deimling [5] that $F(T) \neq \varnothing$.

We now prove the conclusion of the theorem.

First suppose that $u_{n} \rightarrow x^{*}, x^{*} \in F(T)$. Then

$$
\left\|T u_{n}-u_{n}\right\| \rightarrow 0 \text { as } n \rightarrow \infty .
$$

Also, $\left\|u_{n}-u_{n+1}\right\| \rightarrow 0$ as $n \rightarrow \infty$. Again by using the uniform continuity of $T$, we get

$$
\left\|T u_{n}-T u_{n+1}\right\| \rightarrow 0 \text { as } n \rightarrow \infty
$$

Write

$$
\begin{aligned}
\rho_{n}=\left\|x_{n}-u_{n}\right\|, \lambda_{n}= & k \alpha_{n}, \\
\sigma_{n}= & (4-k) \alpha_{n}^{2} D+(2-k) \alpha_{n}^{2}\left\|u_{n}-T u_{n}\right\| \\
& +\alpha_{n}\left[\left\|T x_{n+1}-T y_{n}\right\|+\left\|T u_{n+1}-T u_{n}\right\|\right], \text { and }
\end{aligned}
$$

$\gamma_{n}=2 c_{n} l_{n} D$ in $(2.1 .7)$, we get

$$
\rho_{n+1} \leq\left(1-\lambda_{n}\right) \rho_{n}+\sigma_{n}+\gamma_{n}
$$

From (2.1.8), (2.1.9) and (2.1.10), we have $\frac{\sigma_{n}}{\lambda_{n}} \rightarrow 0$ as $n \rightarrow \infty$, so that $\sigma_{n}=o\left(\lambda_{n}\right)$ and $\Sigma \gamma_{n}<\infty$. Now on using Lemma 1.5, we get

$$
\lim _{n \rightarrow \infty} \rho_{n}=0 .
$$


Since $u_{n} \rightarrow x^{*}$ and from (2.1.11), we have

$$
\left\|x_{n}-x^{*}\right\| \leq\left\|x_{n}-u_{n}\right\|+\left\|u_{n}-x^{*}\right\| \rightarrow 0 \text { as } n \rightarrow 0
$$

which implies $x_{n} \rightarrow x^{*}$ as $n \rightarrow \infty$.

Conversely suppose that $x_{n} \rightarrow x^{*}$ as $n \rightarrow \infty$. Then

$$
\begin{aligned}
\left\|u_{n}-u_{n+1}\right\| & \leq \alpha_{n}\left\|u_{n}-T u_{n}\right\|+c_{n}\left\|T u_{n}-e_{n}\right\| \\
& \leq\left(\alpha_{n}+c_{n}\right) D \rightarrow 0 \text { as } n \rightarrow \infty .
\end{aligned}
$$

Thus by the uniform continuity of $T$, we get

$$
\left\|T u_{n}-T u_{n+1}\right\| \rightarrow \infty \text { as } n \rightarrow \infty .
$$

Now, write

$$
\begin{aligned}
& \rho_{n}=\left\|x_{n}-u_{n}\right\|, \quad \lambda_{n}=k \alpha_{n}, \\
& \sigma_{n}=(6-2 k) \alpha_{n}^{2} D+\alpha_{n}\left[\left\|T x_{n+1}-T y_{n}\right\|+\left\|T u_{n+1}-T u_{n}\right\|\right] \text { and } \\
& \gamma_{n}=2 c_{n} l_{n} D, \text { in }(2.1 .7) \text {, we get } \\
& \qquad \rho_{n+1} \leq\left(1-\lambda_{n}\right) \rho_{n}+\sigma_{n}+\gamma_{n}, \quad n=1,2,3, \ldots
\end{aligned}
$$

From (2.1.12) and (2.1.8), we have

$\sigma=o\left(\lambda_{n}\right)$ and $\Sigma \gamma_{n}<\infty$. Thus by using Lemma 1.5, we get

$\left\|x_{n}-u_{n}\right\| \rightarrow 0$ as $n \rightarrow \infty$.

Since $x_{n} \rightarrow x^{*}$, we have

$\left\|u_{n}-x^{*}\right\| \leq\left\|x_{n}-u_{n}\right\|+\left\|x_{n}-x^{*}\right\| \rightarrow 0$ as $n \rightarrow \infty$ which implies that $u_{n} \rightarrow x^{*}$ as $n \rightarrow \infty$.

This completes the proof of the theorem.

Remarks 2.2.

(i) Theorem 2.1 is proved under the assumption that $T$ is uniformly continuous, by relaxing the Lipschitz property of $T$ in Theorem 1.2.

(ii) From Theorem 2.1, it follows that Theorem 1.3 and Theorem 1.4 are equivalent, provided the initial points of the iterations are same.

Theorem 2.3. Let $K$ be a closed and convex subset of $E$. Assume that $T: K \rightarrow K$ is uniformly continuous and strongly pseudocontractive map with $k \in(0,1)$ with $T(K)$ bounded. For $x_{0}, u_{0}, p_{0}, q_{0}$ and $e_{0}$ in $K$, we define the sequence $\left\{u_{n}\right\}_{n=0}^{\infty}$ and $\left\{x_{n}\right\}_{n=0}^{\infty}$ iteratively by $x_{0}=u_{0} \in K$ as (2.1.1) and (2.1.2) respectively, where $\left\{p_{n}\right\},\left\{q_{n}\right\}$ and $\left\{e_{n}\right\}$ are bounded sequences in $K$ and $\left\{a_{n}\right\},\left\{b_{n}\right\},\left\{c_{n}\right\},\left\{a_{n}^{\prime}\right\},\left\{b_{n}^{\prime}\right\}$ and $\left\{c_{n}^{\prime}\right\}$ are sequences in $(0,1)$ satisfying (i) - (iv) of Theorem 2.1. Then the following are equivalent:

(1) The Mann iteration with modified errors $\left\{u_{n}\right\}$ defined by (2.1.1) converges to $x^{*} \in$ $F(T)$. 
(2) The Ishikawa iteration with modified errors $\left\{x_{n}\right\}$ defined by (2.1.2) converges to $x^{*} \in F(T)$.

Proof. By Deimling [5], $F(T) \neq \varnothing$.

Write

$$
M_{2}=\sup _{n \geq 1}\left\|T y_{n}-p_{n}\right\| \text { and } M_{3}=\sup _{n \geq 1}\left\|T u_{n}-e_{n}\right\| .
$$

From (2.1.6), we have

$$
\begin{aligned}
\left\|x_{n}-u_{n}\right\| \leq & \left(1-k \alpha_{n}\right)\left\|x_{n}-u_{n}\right\|+(3-k) \alpha_{n}^{2}\left\|x_{n}-T y_{n}\right\| \\
& +(2-k) \alpha_{n}^{2}\left\|u_{n}-T u_{n}\right\|+\alpha_{n}^{2}\left\|u_{n}-T y_{n}\right\| \\
& +\alpha_{n}\left[\left\|T x_{n+1}-T y_{n}\right\|+\left\|T u_{n+1}-T u_{n}\right\|\right]+c_{n} l_{n}\left(M_{2}+M_{3}\right)
\end{aligned}
$$

Let

$$
M=\left\|x_{0}-u_{0}\right\|+\sup _{n \geq 1}\left\{\left\|T y_{n}-T u_{n}\right\|+\left\|T y_{n}-p_{n}\right\|+\left\|T u_{n}-e_{n}\right\|\right\}
$$

Obviously $0 \leq M<\infty$. $M$.

Clearly $\left\|x_{0}-u_{0}\right\| \leq M$. Suppose that $\left\|x_{n}-u_{n}\right\| \leq M$. We prove that $\left\|x_{n+1}-u_{n+1}\right\| \leq$

From (2.1.1) and (2.1.2), we have

$$
\begin{aligned}
\left\|x_{n+1}-u_{n+1}\right\| & \leq\left(1-\alpha_{n}\right)\left\|x_{n}-u_{n}\right\|+\alpha_{n}\left[\left\|T y_{n}-T u_{n}\right\|+c_{n}\left[\left\|T y_{n}-p_{n}\right\|+\left\|T u_{n}-e_{n}\right\|\right]\right. \\
& \leq\left(1-\alpha_{n}\right)\left\|x_{n}-u_{n}\right\|+\alpha_{n}\left[\left\|T y_{n}-T u_{n}\right\|+\left\|T y_{n}-p_{n}\right\|+\left\|T y_{n}-e_{n}\right\|\right] \\
& \leq\left(1-\alpha_{n}\right) M+\alpha_{n} M=M
\end{aligned}
$$

Hence

$$
\left\|x_{n}-u_{n}\right\| \leq M \text { for all } n \in N
$$

From (2.1.1) and (2.1.2), we have

$$
\begin{aligned}
\left\|x_{n+1}-y_{n}\right\| \leq & \left(\beta_{n}-\alpha_{n}\right)\left\|x_{n}\right\|+\alpha_{n}\left\|T y_{n}\right\|+\beta_{n}\left\|T x_{n}\right\| \\
& +c_{n}\left\|T y_{n}-p_{n}\right\|+c_{n}^{\prime}\left\|T x_{n}-q_{n}\right\|
\end{aligned}
$$

First we suppose that $u_{n} \rightarrow x^{*}$. It is clear that from (2.3.2), the sequence $\left\{x_{n}\right\}$ is bounded. Then

$$
\begin{aligned}
\left\|x_{n+1}-y_{n}\right\| \leq & \left(\beta_{n}-\alpha_{n}\right)\left\|x_{n}\right\|+\alpha_{n}\left\|T y_{n}\right\|+\beta_{n}\left\|T x_{n}\right\| \\
& +c_{n}\left\|T y_{n}-p_{n}\right\|+c_{n}^{\prime}\left\|T x_{n}-q_{n}\right\| \rightarrow 0 \text { as } n \rightarrow \infty .
\end{aligned}
$$

Now by using the uniform continuity of $T$, we get

$$
\left\|T x_{n+1}-T y_{n}\right\| \rightarrow 0 \text { as } n \rightarrow \infty \text {. }
$$


Since $u_{n} \rightarrow x^{*}$, clearly $\left\|u_{n}-u_{n+1}\right\| \rightarrow 0$ as $n \rightarrow \infty$.

Again by using the uniform continuity of $T$, we get

$$
\left\|T u_{n}-T u_{n+1}\right\| \rightarrow 0 \text { as } n \rightarrow \infty .
$$

Now

$$
\begin{aligned}
\left\|x_{n}-T y_{n}\right\| & \leq\left\|x_{n}-u_{n}\right\|+\left\|u_{n}-T u_{n}\right\|+\left\|T u_{n}-T y_{n}\right\| \\
& \leq\left\|x_{n}-u_{n}\right\|+\left\|u_{n}-T u_{n}\right\|+D_{1},
\end{aligned}
$$

where $D_{1}$ is the diameter of $T(K)$, and

$$
\left\|T y_{n}-u_{n}\right\| \leq\left\|T y_{n}-T u_{n}\right\|+\left\|T u_{n}-u_{n}\right\| .
$$

On substituting (2.3.6) and (2.3.7) in (2.3.1), we get

$$
\begin{aligned}
\left\|x_{n}-u_{n}\right\| \leq & \left(1-k \alpha_{n}\right)\left\|x_{n}-u_{n}\right\|+(3-k) \alpha_{n}^{2}\left[\left\|x_{n}-u_{n}\right\|+\left\|u_{n}-T u_{n}\right\|+D_{1}\right] \\
& +(2-k) \alpha_{n}^{2}\left\|u_{n}-T u_{n}\right\|+\alpha_{n}^{2}\left[\left\|T y_{n}-T u_{n}\right\|+\left\|u_{n}-T u_{n}\right\|\right] \\
& +\alpha_{n}\left[\left\|T x_{n+1}-T y_{n}\right\|+\left\|T u_{n+1}-T u_{n}\right\|\right] \\
& +c_{n}\left[1+(2-k) \alpha_{n}\right]\left[\left\|T y_{n}-p_{n}\right\|+\left\|T u_{n}-e_{n}\right\|\right] \\
\leq & \left(1-k \alpha_{n}+(3-k) \alpha_{n}^{2}\right)\left\|x_{n}-u_{n}\right\|+(6-2 k) \alpha_{n}^{2}\left\|u_{n}-T u_{n}\right\| \\
& +(4-k) \alpha_{n}^{2} D_{1}+\alpha_{n}\left[\left\|T x_{n+1}-T y_{n}\right\|+\left\|T u_{n+1}-T u_{n}\right\|\right] \\
& +c_{n} l_{n}\left(M_{2}+M_{3}\right) .
\end{aligned}
$$
Thus

Since $\alpha_{n} \rightarrow 0$ as $n \rightarrow \infty$, there exists $n_{0} \in N$ such that $\alpha_{n} \leq \frac{5 k}{8(3-k)}$ for all $n \geq n_{0}$.

$$
1-k \alpha_{n}+(3-k) \alpha_{n}^{2} \leq 1-\frac{3 k \alpha_{n}}{8} \quad \text { for all } n \geq n_{0} .
$$

Hence from (2.3.8) and (2.3.9), we have

$$
\begin{aligned}
\left\|x_{n+1}-u_{n+1}\right\| \leq & \left(1-\frac{3 k \alpha_{n}}{8}\right)\left\|x_{n}-u_{n}\right\|+(6-2 k) \alpha_{n}^{2}\left\|u_{n}-T u_{n}\right\|+(4-k) \alpha_{n}^{2} D_{1} \\
& +\alpha_{n}\left[\left\|T x_{n+1}-T y_{n}\right\|+\left\|T u_{n+1}-T u_{n}\right\|\right] \\
& +c_{n} l_{n}\left(M_{2}+M_{3}\right) \quad \text { for all } n \geq n_{0} .
\end{aligned}
$$

Now by applying Lemma 1.5 with

$$
\begin{aligned}
& \rho_{n}=\left\|x_{n}-u_{n}\right\|, \quad \lambda_{n}=\frac{3 k \alpha_{n}}{8}, \\
& \sigma_{n}=(6-2 k) \alpha_{n}^{2}\left\|T u_{n}-u_{n}\right\|+(4-k) \alpha_{n}^{2} D_{1} \\
&+\alpha_{n}\left[\left\|T x_{n+1}-T y_{n}\right\|+\left\|T u_{n+1}-T u_{n}\right\|\right], \quad \text { and } \\
& \gamma_{n}= c_{n} l_{n}\left(M_{2}+M_{3}\right), \text { we get } \sigma_{n}=o\left(\lambda_{n}\right) \text { and } \gamma_{n}<\infty, \text { and } \\
&\left\|x_{n}-u_{n}\right\| \rightarrow 0 \text { as } n \rightarrow \infty, \text { which implies } x_{n} \rightarrow x^{*} \text { as } n \rightarrow \infty .
\end{aligned}
$$


Conversely suppose that $x_{n} \rightarrow x^{*}$ as $n \rightarrow \infty$.

Then by using (2.1.1), we get

$$
\begin{aligned}
\left\|u_{n+1}-u_{n}\right\| & \leq \alpha_{n}\left\|T u_{n}-u_{n}\right\|+c_{n}\left\|T u_{n}-e_{n}\right\| \\
& \leq \alpha_{n}\left(\left\|T u_{n}\right\|+\left\|u_{n}\right\|\right)+c_{n}\left\|T u_{n}-q_{n}\right\| \rightarrow 0 \text { as } n \rightarrow \infty .
\end{aligned}
$$

Thus by the uniform continuity of $T$, we have

$$
\left\|T u_{n}-T u_{n+1}\right\| \rightarrow \infty \text { as } n \rightarrow \infty .
$$

We use the following estimates:

$$
\begin{gathered}
\left\|x_{n}-T y_{n}\right\| \leq\left\|x_{n}-T x_{n}\right\|+\left\|T x_{n}-T y_{n}\right\| \\
\left\|u_{n}-T u_{n}\right\| \leq\left\|u_{n}-x_{n}\right\|+\left\|x_{n}-T x_{n}\right\|+\left\|T x_{n}-T u_{n}\right\| \\
\left\|u_{n}-T y_{n}\right\| \leq\left\|u_{n}-T u_{n}\right\|+\left\|T u_{n}-T y_{n}\right\| \\
\leq\left\|x_{n}-u_{n}\right\|+\left\|x_{n}-T x_{n}\right\| \\
+\left\|T x_{n}-T u_{n}\right\|+\left\|T u_{n}-T y_{n}\right\| .
\end{gathered}
$$

On substituting $(2.3 .12),(2.3 .13)$ and $(2.3 .14)$ in $(2.3 .10)$, we get

$$
\begin{aligned}
\left\|x_{n+1}-u_{n+1}\right\| \leq & \left(1-k \alpha_{n}+(3-k) \alpha_{n}^{2}\right)\left\|x_{n}-u_{n}\right\|+(6-2 k) \alpha_{n}^{2}\left\|x_{n}-T x_{n}\right\| \\
& +(7-2 k) \alpha_{n}^{2} D_{1}+\alpha_{n}\left[\left\|T x_{n+1}-T y_{n}\right\|+\left\|T u_{n+1}-T u_{n}\right\|\right] \\
& +c_{n} l_{n}\left(M_{1}+M_{2}\right) \\
\leq & \left(1-\frac{3 k \alpha_{n}}{8}\right)\left\|x_{n}-u_{n}\right\|+(6-2 k) \alpha_{n}^{2}\left\|x_{n}-T x_{n}\right\| \\
& +(7-2 k) \alpha_{n}^{2} D_{1}+\alpha_{n}\left\|T x_{n+1}-T y_{n}\right\|+\left\|T u_{n+1}-T u_{n}\right\| \\
& +c_{n} l_{n}\left(M_{1}+M_{2}\right) \quad \text { for all } n \geq n_{0} .
\end{aligned}
$$

Write

$$
\begin{aligned}
\rho_{n}= & \left\|x_{n}-u_{n}\right\|, \quad \lambda_{n}=\frac{3 k \alpha_{n}}{8} \\
\sigma_{n}= & (6-2 k) \alpha_{n}^{2}\left\|x_{n}-T x_{n}\right\|+(7-2 k) \alpha_{n}^{2} D_{1} \\
& +\alpha_{n}\left[\left\|T x_{n+1}-T y_{n}\right\|+\left\|T u_{n+1}-T u_{n}\right\|\right], \quad \text { and } \\
\gamma_{n}= & c_{n} l_{n}\left(M_{2}+M_{3}\right) .
\end{aligned}
$$

Here we observe that $\sigma_{n}=o\left(\lambda_{n}\right)$.

Now on using Lemma 1.5, we get

$\left\|x_{n}-u_{n}\right\| \rightarrow 0$ as $n \rightarrow \infty$, which implies $u_{n} \rightarrow x^{*}$ as $n \rightarrow \infty$.

This completes the proof of the theorem. 


\section{Remarks 2.4.}

(i) In Theorem 2.3, we assumed that the range of $T$ is bounded whereas in Theorem 1.2, Soltuz 14] assumed that both the domain and range of $T$ are bounded. Thus, boundedness of the domain of $T$ of Theorem 1.2 is redundant.

(ii) Theorem 2.3 generalizes Theorem 1.2 in the sense that we relaxed the boundedness assumption on the set $K$ and the Lipschitz property of the map $T$ by the uniform continuity of $T$ in Theorem 2.3 .

Corollary 2.5. Let $E$ be a real Banach space and $A: E \rightarrow E$ be a uniformly continuous and strongly accretive map with the range of $(I-A)$ is bounded. For a fixed $f \in E$, define $A^{*}: E \rightarrow E$ by $A^{*} x=f+x-A x$ for each $x \in E$. For a given $x_{0}, p_{0}, q_{0}$, $u_{0}$ and $e_{0} \in E$, we define the sequences $\left\{u_{n}\right\}_{n=0}^{\infty}$ and $\left\{x_{n}\right\}_{n=0}^{\infty}$ iteratively by

$$
u_{n+1}=a_{n} u_{n}+b_{n} A^{*} u_{n}+c_{n} e_{n}, \quad n=0,1,2,3, \ldots
$$

and

$$
\begin{aligned}
x_{n+1} & =a_{n} x_{n}+b_{n} A^{*} y_{n}+c_{n} p_{n}, \\
y_{n} & =a_{n}^{\prime} x_{n}+b_{n}^{\prime} A^{*} x_{n}+c_{n}^{\prime} q_{n}, \quad n=0,1,2, \ldots
\end{aligned}
$$

where $\left\{p_{n}\right\},\left\{q_{n}\right\}$ and $\left\{e_{n}\right\}$ are arbitrary bounded sequences in $K$ and $\left\{a_{n}\right\},\left\{b_{n}\right\},\left\{c_{n}\right\}$, $\left\{a_{n}^{\prime}\right\},\left\{b_{n}^{\prime}\right\}$ and $\left\{c_{n}^{\prime}\right\}$ are sequences in $(0,1)$ satisfying (i) - (iv) of Theorem 2.1 with $x_{0}=u_{0}$ in $E$, then the following are equivalent:

(1) The Mann iteration with modified errors (2.5.1) converges to the solution of the equation $A x=f$.

(2) The Ishikawa iteration with modified errors (2.5.2) converges to the solution of the equation $A x=f$.

Proof. Existence of the solution follows from Deimling 5]. Since $A$ is uniformly continuous it follows that $A^{*}$ is uniformly continuous. Since the range of $(I-A)$ is bounded, we have $\left\|f+(I-A) x_{n}\right\|$ is bounded and also $\left\|f+(I-A) y_{n}\right\|$ is bounded. Hence Theorem 2.3 gives the conclusion of Corollary 2.5.

Corollary 2.6. Let $E$ be a real Banach space and $T: E \rightarrow E$ a uniformly continuous and strongly accretive map. Suppose the range of $T$ is bounded. For a fixed $f \in E$, define $S: E \rightarrow E$ by $S x=f-T x$ for each $x \in E$. For a given $x_{0}, p_{0}, q_{0}, u_{0}$ and $e_{0} \in E$, we define the sequences $\left\{u_{n}\right\}_{n=0}^{\infty}$ and $\left\{x_{n}\right\}_{n=0}^{\infty}$ iteratively by

$$
\begin{aligned}
u_{n+1} & =a_{n} u_{n}+b_{n} S u_{n}+c_{n} e_{n}, \quad n=0,1,2,3, \ldots \\
x_{n+1} & =a_{n} x_{n}+b_{n} S y_{n}+c_{n} p_{n}, \\
y_{n} & =a_{n}^{\prime} x_{n}+b_{n}^{\prime} S x_{n}+c_{n}^{\prime} q_{n}, \quad n=0,1,2, \ldots
\end{aligned}
$$

where $\left\{p_{n}\right\},\left\{q_{n}\right\}$ and $\left\{e_{n}\right\}$ are arbitrary bounded sequences in $K$ and $\left\{a_{n}\right\},\left\{b_{n}\right\},\left\{c_{n}\right\}$, $\left\{a_{n}^{\prime}\right\},\left\{b_{n}^{\prime}\right\}$ and $\left\{c_{n}^{\prime}\right\}$ are sequences in $(0,1)$ satisfying (i) - (iv) of Theorem 2.1 with $x_{0}=u_{0}$ in $E$, then the following are equivalent: 
(1) The Mann iteration with modified errors (2.6.1) converges to the solution of the equation $x+T x=f$.

(2) The Ishikawa iteration converges with modified errors (2.6.2) to the solution of the equation $x+T x=f$.

Proof. We define $A=I+T$, then clearly $A$ is uniformly continuous and strongly accretive. Hence on using Deimling's result [6], the equation $A x=f$ has a unique solution, say $x^{*}$ in $E$. Since the range of $T$ is bounded it follows that range of $I-A$ is bounded. Also,

$$
S x=f-T x=f-(A-I) x=f+(I-A) x=A^{*} x,
$$

where $A^{*}$ is as defined in Corollary 2.5. Now from Corollary 2.5, the conclusion follows.

\section{Acknowledgements}

This work is supported by U. G. C. Major Research Projece Grang No. F. 8-8/2003 (SR). The authors thank the University Grants Commission, India, for the financial support.

\section{References}

[1] J. Bogin, On strict pseudocontractions and a fixed point theorem, Technian preprint series no: MT-219, Haifa, Israel, 1974.

[2] C. E. Chidume, Iterative approximation of fixed points of Lipschitz strictly pseudocontractive mappings, Proc. Amer. Math. Soc. 99(1987), 283-288.

[3] C. E. Chidume, Convergence theorems for strongly pseudocontractive and stongly accretie maps, J. Math. Anal. Appl. 228(1998), 254-264.

[4] C. E. Chidume and M. O. Osilike, Nonlinear accretive and pseudocontractive operator equations in Banach spaces, Nonlinear Anal. TMA 31(1998), 779-789.

[5] K. Deimling, Zeros of Accretive operators, Manuscripta Math. 13(1974), 365-374.

[6] K. Deimling, Nonlinear Functional Analysis, Springer - Verlag, 1985.

[7] Z. Hiyein and Y. Yuting, Approximation of fixed points of strongly pseudocontractive maps without Lipschitz assumption, Proc. Amer. Math. Soc. 125(1997), 1705-1709.

[8] S. Ishikawa, Fixed point by a new iteration method, Proc. Amer. Math. Soc. 44(1974), 147-150.

[9] T. Kato, Nonlinear semigroups and evolution equations, J. Math. Soc. Japan 19(1967), 508-520.

[10] L. S. Liu, Ishikawa and Mann iteration process with errors for nonlinear stongly accretive mappings in Banach spaces, J. Math. Anal. Appl. 194(1995), 114-125.

[11] W. R. Mann, Mean value methods in iteration, Proc. Amer. Math. Soc. 4(1953), 506-510.

[12] B. E. Rhoades and S. Soltuz, On the equivalence of Ishikawa and Mann iteration methods, Int. J. Math. Math. Sci. 7(2003), 451-459. 
[13] K. P. R. Sastry and G. V. R. Babu, Approximation of fixed points of strongly pseudocontractive mappings on arbitrary closed, convex sets in a Banach space, Proc. Amer. Math. Soc. 128(2000).

[14] Stefan M Soltuz, The convergence of Mann iteration with errors is equivalent to the convergence of Ishikawa iteration with errors, Lecturas Matemátics 25(2004), 5-15.

[15] Y. Xu, Ishikawa and Mann iterative processes with errors for nonlinear strongly accretive operator equations, J. Math. Anal. Appl. 224(1998), 91-101.

Department of Mathematics, Andhra University, Visakhapatnam - 530 003, India.

E-mail: gvr_babu@hotmail.com

Department of Mathematics, Andhra University, Visakhapatnam - 530 003, India.

E-mail: kameswaridvr@yahoo.co.in 